\title{
Taking Responsibility: Psychological and Attitudinal Change through a Domestic Violence Intervention Program in New South Wales, Australia
}

\author{
Timothy R. Broady ${ }^{1}$, Rebecca M. Gray ${ }^{1}$ \\ ${ }^{1}$ Relationships Australia NSW, Sydney, Australia \\ Correspondence: Timothy Broady, Relationships Australia NSW, P. O. Box 371, North Ryde BC, NSW 1670, Australia.
}

Received: April 5, 2017

Accepted: May 2, $2017 \quad$ Available online: May 22, 2017

doi:10.11114/ijsss.v5i6.2321

URL: https://doi.org/10.11114/ijsss.v5i6.2321

\begin{abstract}
Literature widely reports the negative impacts of domestic violence at individual, family, and societal levels. Intervention programs that effectively assist violent men to develop alternate ways of relating, and thus enhance the safety of women and children, are of significant value to governments and the community. This study evaluates the effectiveness of one such program in promoting change in relevant attitudes and psychological constructs. Program participants completed pre- and post-group surveys containing validated scales that measured their gender equity beliefs, self-esteem, mastery, and psychological distress. Over the duration of program attendance, positive changes were evident regarding men's self-esteem, mastery, and psychological distress, however, no significant change in gender equity beliefs was apparent. The positive changes evident amongst participants indicate beneficial outcomes from group work participation in areas that have been identified as risk factors for violent behaviour. The results also suggest that intervention programs would benefit from an increased focus on gender equity beliefs, and that further research is necessary on the extent to which this focus could improve attitudes, and consequently promote safety for women and children.
\end{abstract}

Keywords: domestic violence, gender equity, men's behaviour change program, perpetrators, group work

\section{Introduction}

\subsection{Prevalence and Impact of Domestic Violence}

More than 29,000 incidents of domestic violence related assault were recorded across New South Wales (NSW) over the 12 months up to September, 2016, with the prevalence of reported incidents remaining stable over the past five years (NSW Bureau of Crime Statistics and Research, 2016). Such prevalence highlights the importance of this social issue in Australia. The significance is further emphasised by recent figures from the Australian Bureau of Statistics, who estimate that $17 \%$ of Australian women have experienced violence from intimate partners in their lifetime (ABS, 2012).

The impacts of domestic violence have been widely reported, particularly in terms of victims' physical health, and potentially more serious implications for their emotional and psychological wellbeing (Jordan, Campbell, \& Follingstad, 2010; Sety, 2011; Tarzia et al., 2016; Thomas \& Scott-Tilley, 2017). Due to the major consequences of domestic violence on individuals, families, and communities, intervention practices that assist in the reduction of violent behaviour are of significant value. In attempting to reduce the impact and prevalence of domestic violence, the need to engage with both those affected by and those who use violence has become increasingly recognised (Campbell, Neil, Jaffe, \& Kelly, 2010; Day, O'Leary, Chung, \& Justo, 2009).

\subsection{Domestic Violence Intervention Programs}

In Australia, intervention programs for men who have used violence towards their partners have become common, though the existing evidence regarding the effectiveness of these programs (both domestically and internationally) has proven somewhat inconclusive, mainly due to methodological inconsistencies and practical constraints in conducting rigorous evaluations (Australian Attorney-General's Department, 2010; Heseltine, Day, \& Sarre, 2011). The primary focus of many evaluation studies has been the extent of repeated physical assault or rates of criminal arrest after program completion, which is considered to be a narrow view of program effectiveness (Mackay, Gibson, Lam, \& Beecham, 2015; Tutty \& Babins-Wagner, in press). Meta-analytic review of such studies has found the treatment effects of interventions are small, indicating that the programs under investigation had minimal effect in terms of reducing violence beyond the effect of reducing further arrest (Babcock, Green, \& Robie, 2004). In light of this, Hellman, 
Johnson, and Dobson (2010) suggest that it is more appropriate to investigate factors that promote personal change amongst perpetrators of domestic violence, rather than solely focus on recidivism. Similarly, Westmarland and Kelly (2013) argue that measures of success in men's behaviour change programs ought to be extended to include issues such as: improved relationships between men, women, and children; developing respect and effective communication; providing women with space to make their own choices; and improved self-awareness and understanding of the impact of violence on women and children. Intervention program effectiveness is therefore broader than attendees' behavioural change, and measuring effectiveness ought to consider personal and attitudinal change, and relationship dynamics involving the partners and families of program participants (Day et al., 2009; Laing, 2003; Westmarland \& Kelly, 2013).

This article reports on the quantitative phase of a longitudinal, mixed methods research project evaluating the effectiveness of one particular intervention program - "Taking Responsibility". Taking Responsibility is a group-based intervention program run by Relationships Australia NSW which aims to facilitate change amongst men who have behaved violently towards their partners. It is designed to support men to take responsibility for their actions and adopt alternative ways of relating to others. The course includes an 18-week group work component, which is complemented by periodic individual support sessions. Program content covers topics such as: the tactics of power and control; exploring beliefs about gender roles; understanding the impact of violence on others; de veloping empathy for those affected; and exploring thought processes underpinning violent behaviour. The group also utilises mindfulness techniques to promote self-regulation, and several experiential exercises to assist in developing empathy and reflective capacity. This is accompanied by a therapeutic aspect, which is more spontaneous and client-centred, largely undertaken through group discussion. This discussion provides an opportunity to address any arising issues, particularly clients' attempts to apply course material to everyday life.

The first stage of this project identified four key psychological characteristics, outlined below, that have been argued to influence the propensity of men to behave violently towards their partners and their ways of relating to other people more broadly (Broady, Gray, \& Gaffney, 2014). This paper reports on the second stage of the project, which assessed the extent of personal change in relation to these characteristics that occurred over the duration of course participation.

\subsection{Risk Factors for Domestic Violence}

A great deal of literature has identified various risk factors for domestic violence, with a significant proportion referring to external influences, e.g., socioeconomic status, substance use/abuse, childhood experiences of violence (e.g., Hotaling \& Sugarman, 1986; Reingle, Jennings, Connell, Businelle, \& Chartier, 2014; Smith, Homish, Leonard, \& Cornelius, 2012). Since Taking Responsibility aims to develop better ways of relating, the focus of the program is on personal change and equipping participants with skills and tools to respond more positively to external factors. While acknowledging the major influence that external risk factors such as those listed above can have on violent behaviour, this project focuses on investigating personal, intrinsic characteristics that are likely to impact on the interpersonal relationships between group participants and their partners, particularly in relation to violent behaviour. Moreover, men requiring support with external factors will be referred to allied services which specialise in these presenting problems, such as residential rehabilitation. With the context of these external factors in mind, the characteristics investigated in this project are: gender equity, self-esteem, mastery, and psychological distress.

\subsubsection{Gender Equity}

Attitudes relating to gender are significantly related to violence against women with inequitable views of gender roles associated with attitudes supporting the use of such violent behaviour (Dardis, Dixon, Edwards, \& Turchik, 2015; Das et al., 2012; Lei, Simons, Simons, \& Edmond, 2014). Large scale Australian surveys have similarly found a strong relationship between attitudes that are accepting of violence against women and "gender equity scores", where high support for gender equity reflects a belief that women and men have the same entitlements to roles and opportunities in society (McGregor, 2009; Taylor \& Mouzos, 2006; Webster et al., 2014). Furthermore, the first phase of the present study found that men commencing the Taking Responsibility course held significantly lower gender equity beliefs than the general population (Broady et al., 2014). Although there is wide consensus regarding the significance of attitudes towards gender equity, there is scant research examining the extent to which these attitudes are influenced by intervention programs. One notable exception is Gondolf's (2000) multi-site study, which found that despite one in five male program attendees reporting a change in their gender attitudes, overall program objectives of affecting men's attitudes towards women were not met.

\subsubsection{Self-esteem}

Issues of self-esteem have also been found to impact on an individual's propensity to behave abusively towards others, particularly intimate partners. While there is some contention in the literature surrounding the relationship between violence and self-esteem (Walker \& Bright, 2009), it has been suggested that any indications of high self-esteem amongst men who use violence are falsely inflated attempts at masking the opposite reality (Salmivalli, 2001). The 
majority of existing literature suggests that low self-esteem is a risk factor for violent behaviour - a relationship supported by the most empirically rigorous studies (Walker \& Bright, 2009). This is also supported by the first phase of the current project, where men commencing the Taking Responsibility program were found to demonstrate significantly lower self-esteem than general populations (Broady et al., 2014).

\subsubsection{Mastery}

A sense of personal mastery is particularly relevant in current understandings of domestic violence, especially in terms of intervention programs. Many intervention approaches (e.g., the Duluth Model) are based on placing accountability on those who behave violently, and this is also reflected in the Minimum Standards for Men's Domestic Violence Behaviour Change Programs in New South Wales (NSW Attorney General, 2011) and Australia's National Plan to Reduce Violence Against Women and Their Children (COAG, 2011). Existing research suggests that men who have been abusive towards their partners are often characterised by feelings of powerlessness, and their violence represents an attempt to regain control within their relationships (Schmidt, Lisco, Parrott, \& Tharp, 2016; Walker \& Knauer, 2011). This is also supported by the first phase of the current project, with men commencing the Taking Responsibility program reporting lower mastery levels than the general population (Broady et al., 2014).

\subsubsection{Psychological Distress}

The final area of investigation in this study is psychological distress. Higher prevalence of psychological issues (including elevated distress and psychopathology) have been widely reported amongst men who have used domestic violence (Declercq, Willemsen, Audenaert, \& Verhaeghe, 2012; Gibbons, Collins, \& Reid, 2011; Wallinius, Nilsson, Hofvander, Anckarsäter, \& Stålenheim, 2012). The first phase of the present study further supports this, with men commencing the Taking Responsibility course reporting significantly higher levels of psychological distress than those reported in the general population (Broady et al., 2014).

\subsection{Present Study}

Previous literature therefore suggests that men attending domestic violence intervention programs are likely to exhibit low support for gender equity, low self-esteem, low mastery, and high psychological distress. This reflects the profile of men commencing Taking Responsibility (Broady et al., 2014). Any intervention program that effectively addresses these issues may therefore help in assisting men to develop better ways of relating to their partners and children, and subsequently reduce their violent behaviour, thereby enhancing the safety of women and children. This paper investigates the extent to which men demonstrated change in relation to the aforementioned characteristics over the duration of their attendance at the Taking Responsibility course.

\section{Method}

\subsection{Participants}

Men attending Taking Responsibility were invited to participate in the research by completing a short survey at both the beginning and end of the course. Program intake includes a combination of mandated, referred, and self-referred men.

Ethics approval for this study was granted by the Relationships Australia NSW Human Research Ethics Committee.

A total of 130 men from 20 different groups throughout New South Wales agreed to participate. Of the 130 participants, 114 (87.7\%) completed the pre-group survey, and 70 (53.8\%) completed the post-group survey, with 56 (43.1\%) completing both. In order to investigate changes in the characteristics under investigation, only the 56 matched pre-post surveys were included in analysis for this paper.

Acomparison of the demographic profiles of the full sample and those who completed both pre- and post-group surveys is provided in Table 1.

Table 1. Participant demographics

\begin{tabular}{|c|c|c|}
\hline & Full sample $(\mathrm{n}=130)$ & Pre- and post- surveys $(n=56)$ \\
\hline Age & $\mathrm{M}=39.32$ years, $\mathrm{SD}=9.17$ & $\mathrm{M}=41.91$ years, $\mathrm{SD}=8.78$ \\
\hline \multirow[t]{4}{*}{ Income $^{1}$} & Low: $36.2 \%$ & Low: $41.1 \%$ \\
\hline & Medium: $27.7 \%$ & Medium: $30.4 \%$ \\
\hline & High: $23.1 \%$ & High: $23.2 \%$ \\
\hline & Refused: $13.1 \%$ & Refused: $5.4 \%$ \\
\hline \multirow[t]{4}{*}{ Relationship status ${ }^{2}$} & Married: $29.2 \%$ & Married: $37.5 \%$ \\
\hline & De facto: $20.8 \%$ & De facto: $19.6 \%$ \\
\hline & Separated: $26.2 \%$ & Separated: $32.1 \%$ \\
\hline & Other/Refused: $23.8 \%$ & Other/Refused: $10.8 \%$ \\
\hline Living with partner ${ }^{2}$ & $40.0 \%$ & $48.2 \%$ \\
\hline Seeing a counsellor ${ }^{2}$ & $58.5 \%$ & $66.1 \%$ \\
\hline
\end{tabular}


${ }^{1}$ Low $=$ A \$0-599/week; Medium = A\$600-999/week; High = A\$1000+/week

${ }^{2}$ Relationship status, living with partner, and seeing counsellor reported at beginning of course.

Some demographic differences are apparent between those participants who completed both surveys and the full sample. Notably, those who completed both were more likely to have reported living with their partner and seeing a counsellor at the beginning of the course. They were also more likely to have disclosed their relationship status, with higher proportions indicating that they were either married or separated at the beginning of the course.

\subsection{Surveys}

Surveys were distributed amongst group members by a research officer who was not associated with the clinical work of the groups. In order to obtain baseline measures, these surveys were completed within the first two weeks of group attendance. Post-group surveys were then distributed during the final week of the course. Surveys contained demographic questions and validated scales addressing gender equity, self-esteem, mastery, and psychological distress.

\subsubsection{Gender Equity}

Gender equity was measured using the same scale that has been used in the Australian National Community Attitudes Toward Violence Against Women Survey (NCAS) (McGregor, 2009; Webster et al., 2014). This scale includes eight statements regarding equality between men and women, ans wered on a scale of 1 (strongly disagree) to 5 (strongly agree). Following the same procedure as NCAS, responses were summed and converted to a score out of 100, before being categorised as high $(>90)$, medium $(75-90)$, or low $(<75)$ support for gender equity. Reliability of the Gender Equity Scale in this study was $\alpha=.74$ (pre-group) and $\alpha=.83$ (post-group).

\subsubsection{Self-esteem}

Self-esteem was measured using the Rosenberg Self-Esteem Scale (Rosenberg, 1965). This scale consists of ten items, answered on a scale of 1 (strongly disagree) to 4 (strongly agree). Negatively worded items were reverse scored before item scores were summed to provide a score out of 40 , with higher scores indicating higher self-esteem. Reliability in this study was $\alpha=.84$ (pre-group) and $\alpha=.88$ (post-group).

\subsubsection{Mastery}

Mastery was measured using the scale developed by Pearlin and Radabaugh (1976). This scale consists of seven items, answered on a scale of 1 (strongly disagree) to 4 (strongly agree), and item scores summed, with higher scores indicating greater levels of mastery. Scores were then converted into categories based on cut-offs developed by the original authors: limited mastery $(<18)$, moderate mastery $(18-23)$, or great mastery $(>23)$. Reliability in this study was $\alpha=.69$ (pre-group) and $\alpha=.85$ (post-group).

\subsubsection{Psychological Distress}

Distress was measured using the six-item Kessler Scale of Psychological Distress (K6; Kessler et al., 2003). Items were answered on a scale of 1 (none of the time) to 5 (all of the time), indicating the frequency of certain indicators of distress over the past 30 days. Responses were summed, with higher scores indicating higher levels of psychological distress. Scores were then also categorised to reflect participants' distress levels: low $(<13)$, medium (14-18), or high $(>18)$. Reliability of the K6 in this study was $\alpha=.88$ (pre-group) and $\alpha=.92$ (post-group).

\section{Results}

To determine the extent of any changes over time in the course, post-group questionnaire scores were compared with those from pre-group surveys and normative samples. A repeated measures methodology was utilised, thereby only including participants who completed both pre-group and post-group surveys. However, it is worth noting that the pre-group scores of those who did not complete post-group surveys were not significantly different from those who did complete both surveys (see Table 2).

Table 2. Comparison of pre-group questionnaire scores between participants who also completed post-group surveys and those who did not

\begin{tabular}{lccc}
\hline \multicolumn{1}{c}{ Questionnaire } & Pre-group survey only $(\mathrm{n}=58)$ & $\begin{array}{c}\text { Pre- and post-group surveys } \\
(\mathrm{n}=56)\end{array}$ & Sig. \\
\hline Gender Equity Scale & $\mathrm{M}=70.70, \mathrm{SD}=10.82$ & $\mathrm{M}=72.12, \mathrm{SD}=10.81$ & $t_{(112)}=.70, p=.49$ \\
Rosenberg Self-Esteem Scale & $\mathrm{M}=27.70, \mathrm{SD}=4.80$ & $\mathrm{M}=27.64, \mathrm{SD}=3.70$ & $t_{(94)}=.31, p=.94$ \\
Mastery Scale & $\mathrm{M}=20.36, \mathrm{SD}=3.11$ & $\mathrm{M}=20.52, \mathrm{SD}=2.80$ & $t_{(111)}=.22, p=.83$ \\
K6 & $\mathrm{M}=13.65, \mathrm{SD}=5.21$ & $\mathrm{M}=13.42, \mathrm{SD}=4.78$ & $t_{(112)}=.25, p=.80$ \\
\hline
\end{tabular}

3.1 Gender Equity

The mean post-group gender equity score of respondents $(73.67, \mathrm{SD}=12.15)$ was not significantly different from the 
pre-group mean $(72.12, \mathrm{SD}=10.81), t_{(55)}=1.21, p=.23$, and the effect size was small, $d=.16$. The distributions of respondents scores across gender equity categories (low, medium, high) from pre- and post-group surveys were also compared with male respondents from the 2013 NCAS (Webster et al., 2014), as shown in Figure 1.

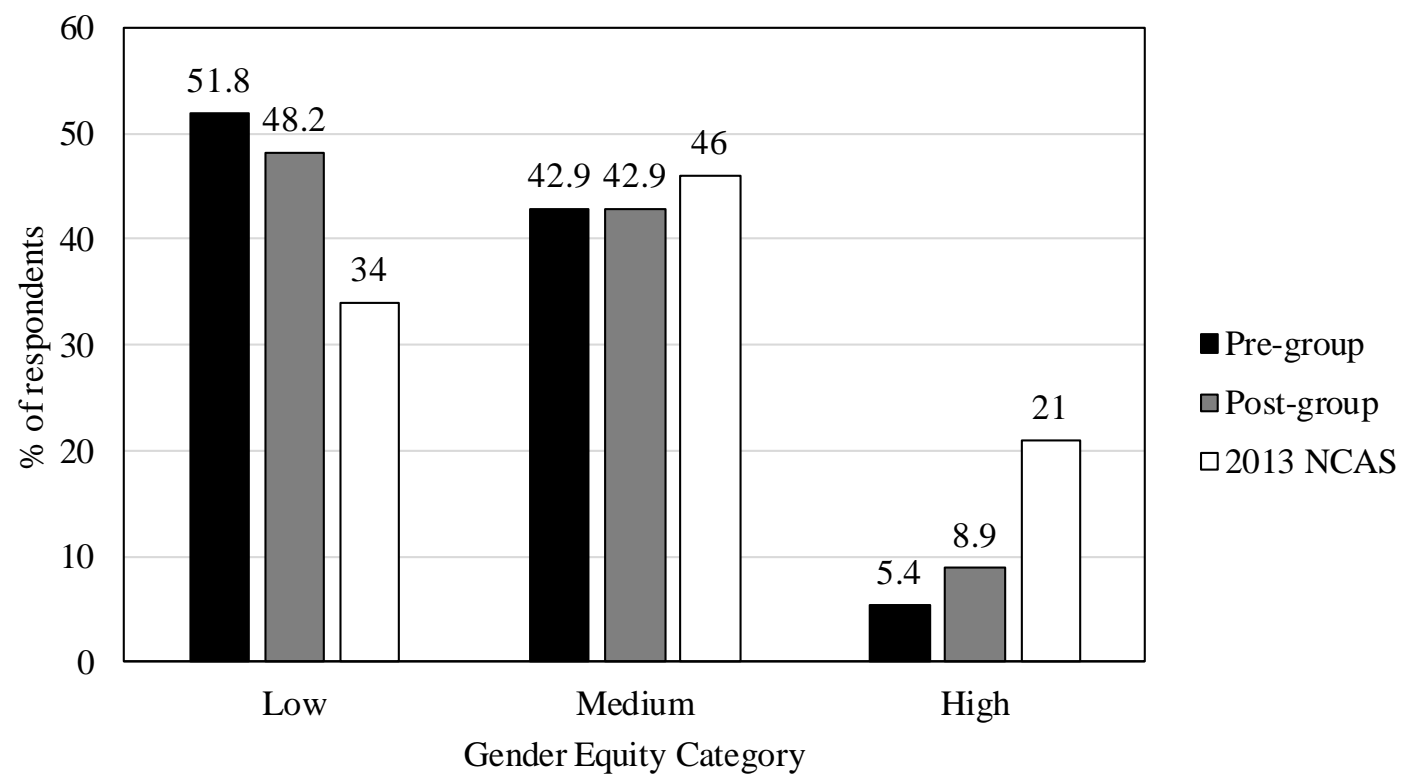

Figure 1. Distribution of participants across gender equity categories

Although the proportion of respondents with low support for gender equity was smaller at post-group than at pre-group, and a larger proportion reported high support, the difference in these distributions was not statistically significant, $\chi_{(2)}^{2}=1.30, p=.52$. Both pre-group and post-group scores demonstrated significantly different distributions to the national NCAS sample $\left(\chi_{(2)}^{2}=14.10, p=.001\right.$, and $\chi_{(2)}^{2}=7.29, p=.03$ respectively), with participants in this study generally categorised as having less support for gender equity than the national sample. The effect sizes of these differences were small to moderate, $\varphi=.26$ (pre-group) and $\varphi=.19$ (post-group).

\subsection{Self-esteem}

Respondents' mean self-esteem score was significantly higher at post-group (30.16, SD=4.15) than pre-group (27.64, $\mathrm{SD}=3.70), t_{(49)}=4.29, p<.001$, with a moderate effect size, $d=.61$. Pre-group and post-group scores were also compared with the RSES scores from an Australian general community sample ( $\mathrm{N}=485, \mathrm{M}=31.07, \mathrm{SD}=5.15$; Schmitt \& Allik, 2005). Pre-group scores from the current study were significantly lower than the general community sample, $t_{(535)}=4.49, p=.003$, with a relatively large effect size, $d=.77$, while the difference between post-group scores and the general community sample was not statistically significant, $t_{(537)}=1.44, p=.14$, with a small effect size, $d=.20$. These comparisons are illustrated in Figure 2. 


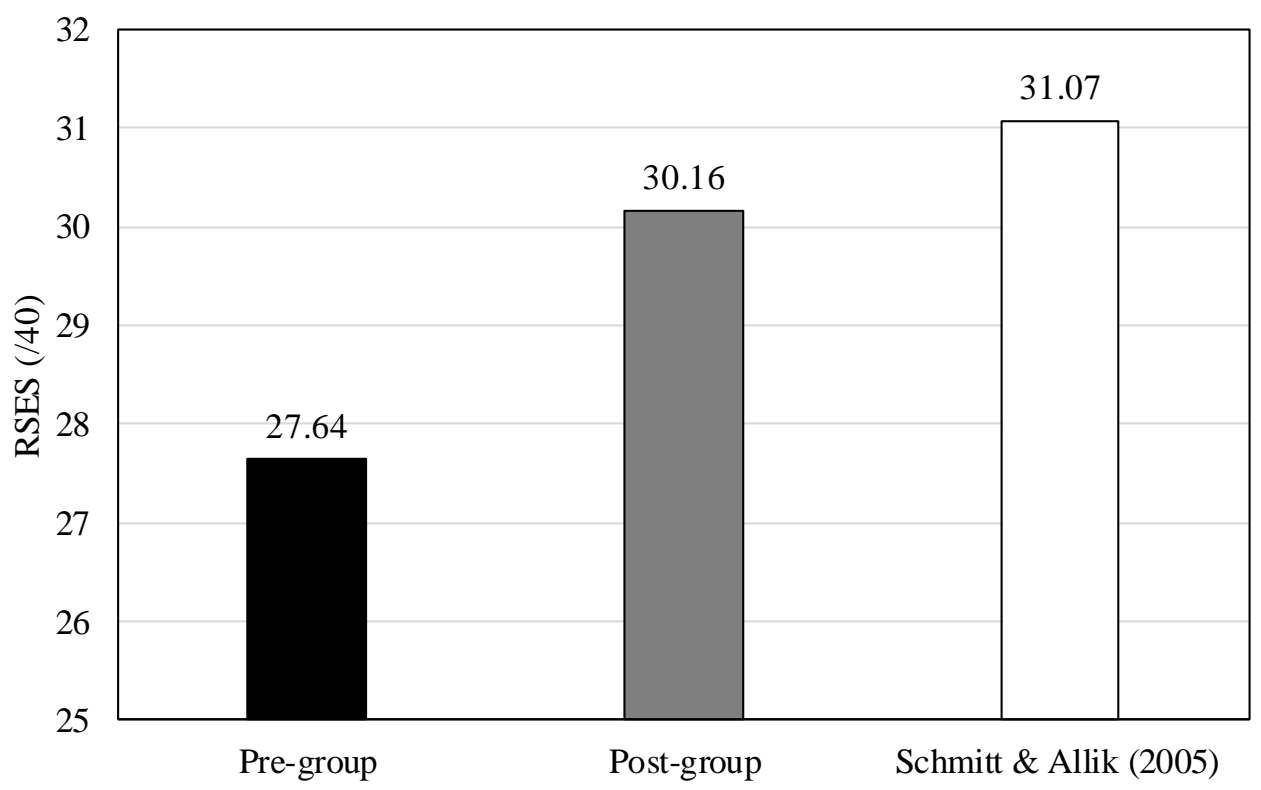

Figure 2. Comparison of self-esteem scores from pre- and post-group and normative Australian sample

\subsection{Mastery}

Mastery scores were significantly higher at post-group $(\mathrm{M}=22.12, \mathrm{SD}=3.23)$ than at pre-group $(\mathrm{M}=20.52, \mathrm{SD}=2.80)$, $t_{(53)}=3.67, p=.001$, with a moderate effect size, $d=.50$. The distributions of respondents across mastery categories (limited, moderate, great) were also compared between pre-group, post-group, and the normative sample initially used in validating the mastery scale (Pearlin \& Radabaugh, 1976), as shown in Figure 3.

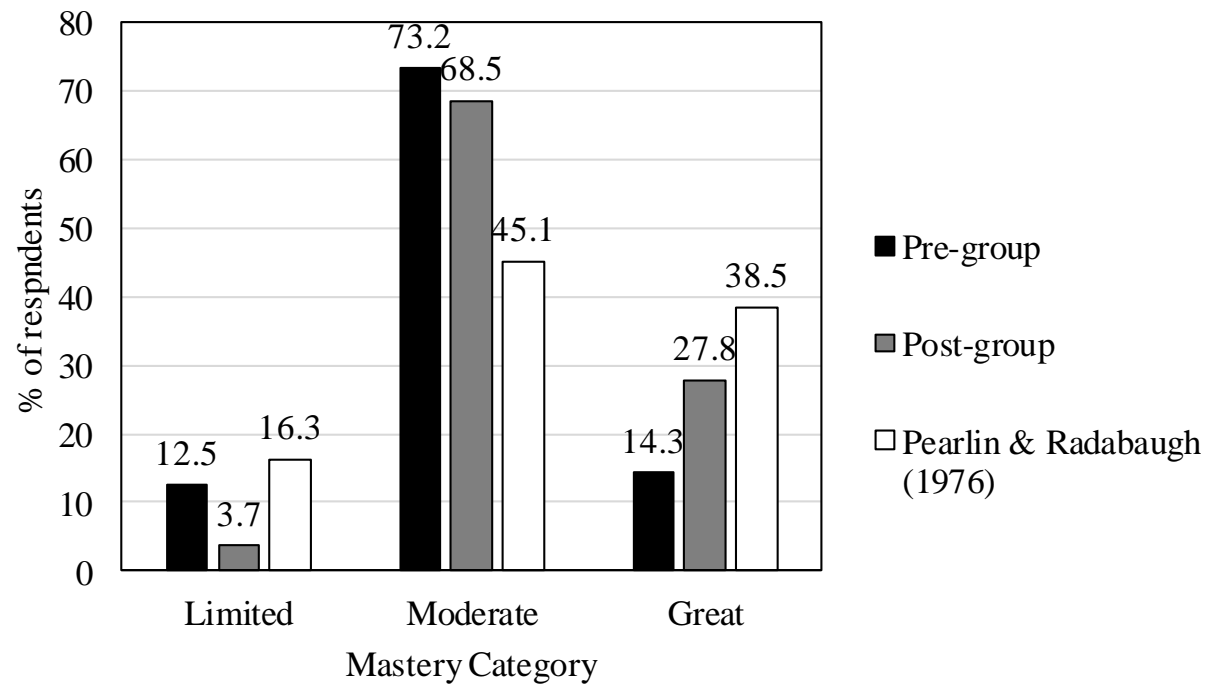

Figure 3. Distribution of participants across mastery categories

The proportion of respondents categorised as having limited or moderate mastery decreased from pre-group to post-group, with a simultaneous increase in the proportion of those displaying great mastery. The difference between these distributions was statistically significant, $\chi_{(2)}^{2}=9.54, p=.01$, though the effect size was relatively small, $\varphi=.22$. Furthermore, both pre-group and post-group results demonstrated a larger proportion of participants categorised as having moderate mastery than the normative sample studied by Pearlin and Radabaugh (1976), with smaller proportions categorised as either limited or great mastery. The differences between the normative sample distribution and both pre-group and post-group distributions were statistically significant $\left(\chi_{(2)}^{2}=18.75, p<.001\right.$, effect size, $\varphi=.31$, and $\chi_{(2)}^{2}=14.05, p=.001$, effect size, $\varphi=.26$, respectively). 


\subsection{Psychological Distress}

K6 scores were significantly lower at post-group $(\mathrm{M}=10.65, \mathrm{SD}=4.37)$ than at pre-group $(\mathrm{M}=13.42, \mathrm{SD}=4.78), t_{(54)}=4.03$, $p<.001$, with a moderate effect size, $d=.54$. The distributions of respondents across psychological distress categories (low, medium, high) were also compared from pre-group and post-group surveys, and a normative population of male Australian employees (Hilton et al., 2008), as shown in Figure 4.

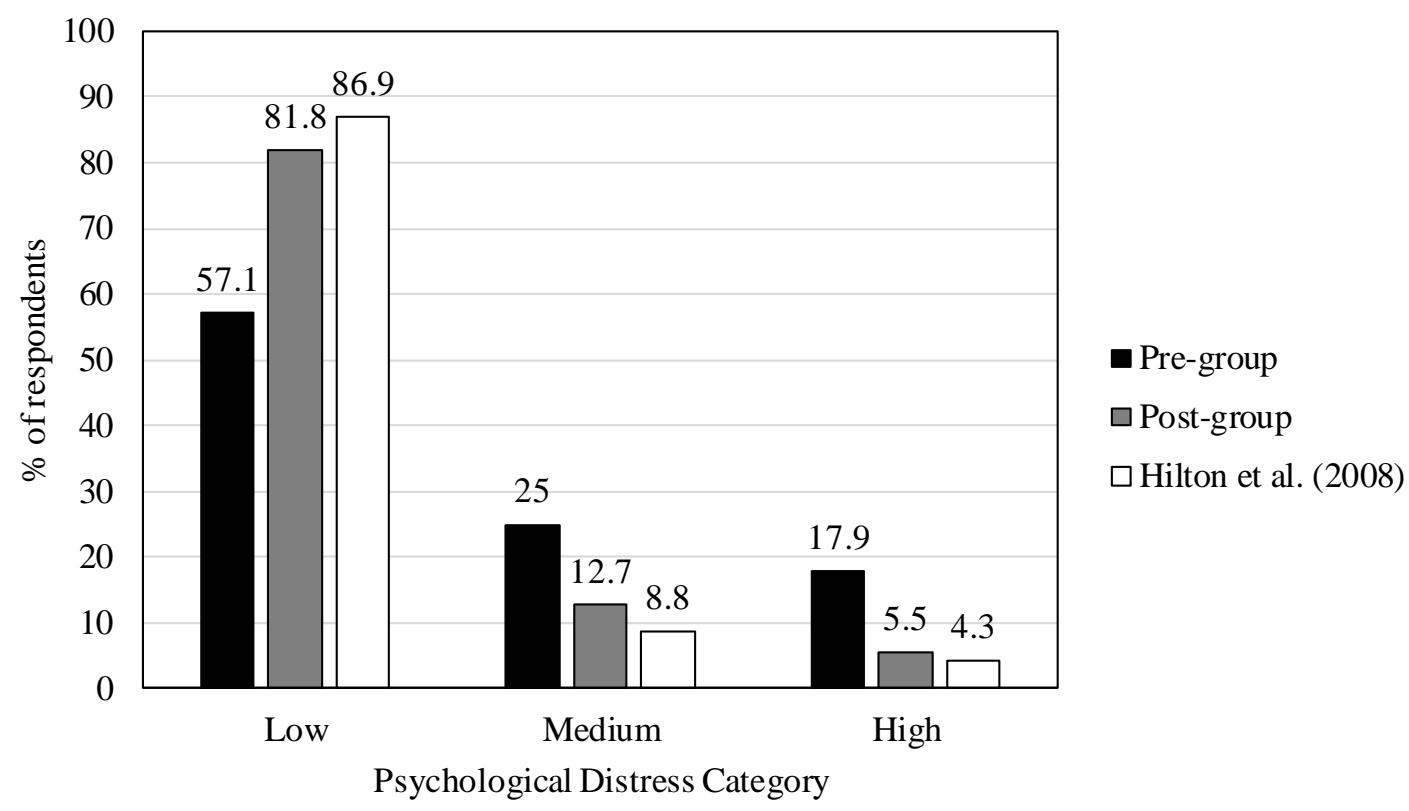

Figure 4. Distribution of participants across psychological distress categories

The proportion of respondents categorised as reporting medium and high le vels of psychological distress decreased from pre-group to post-group, with a corresponding increase in the proportion of respondents who reported low psychological distress. The difference between these distributions was statistically significant, $\chi_{(2)}^{2}=14.28, p=.001$, though the effect size was relatively small, $\varphi=.27$. The pre-group distribution was significantly different from the normative sample, $\chi_{(2)}^{2}=22.69$, $p<.001$, with greater proportions of respondents reporting medium or high levels of psychological distress, and a smaller proportion reporting low distress. The effect size of this difference was moderate, $\varphi=.34$. Importantly, the post-group distribution was not significantly different from the normative sample, $\chi_{(2)}^{2}=1.27, p=.53$, with a very small effect size, $\varphi=.08$.

\section{Discussion}

This repeated measures quantitative phase of a broader evaluation project investigated changes in certain characteristics that have been associated with domestic violence perpetration over the time spent attending a men's group intervention. Having previously identified the significance of gender equity beliefs, self-esteem, mastery, and psychological distress (see Broady et al., 2014), the following positive changes were observed amongst participants over the duration of their program attendance: increased self-esteem, increased mastery, and decreased psychological distress.

While pre-group results indicated heightened experiences of psychological distress (which are argued to increase the likelihood of behaving violently), post-group results suggest no significant differences between the current sample's experience of psychological distress and that of the wider Australian population. These results may therefore indicate that the risk of domestic violence as a result of high distress was minimised through program attendance. Alternatively, these changes in psychological distress scores could be attributed to comfort within the group environment, as opposed to any feelings of apprehension that may have been experienced during early stages of program attendance. Since the K6 is a generalised measure of psychological distress and is time limited (with a focus on the preceding four weeks), it is possible that changes in participants' scores could be the function of any number of other external factors (e.g., changes in social support, outcomes of legal proceedings, etc.). While alternative explanations outside the contribution of the course could contribute to the evident changes, the significance of these changes should not be underestimated. Regardless of the reasons behind the reduction in psychological distress amongst participants, results indicate that participants felt significantly less distressed in the final weeks of the program than they did at the beginning. Considering the previously outlined evidence indicating that psychological distress can increase the propensity to behave violently, these findings 
suggest that participants may be less likely to use violence at the conclusion of the course than they were prior to its commencement.

A similar trend was evident in relation to self-esteem. Compared to normative data, self-esteem scores were low at pre-group, but increased to be in line with norms at post-group. While it is possible that external factors could also have contributed to changes in reported self-esteem, course content specifically addresses issues related to self-perceptions and therefore is likely to have had some degree of influence. Increased self-esteem and positive self-perceptions can be argued to positively influence interpersonal relationships, with a better view of self leading to better ways of relating to others.

Mastery scores were also found to increase from pre-group to post-group, suggesting that participants felt more in control of their own lives at the end of the course. Since course content includes teaching techniques and skills to remain calm and composed instead of losing control, this increase in mastery could well reflect an increased capacity for self-control in emotive situations and therefore reduce the likelihood of reacting violently towards a partner. This is also reflective of the Duluth Model, the Minimum Standards for Domestic Violence Behaviour Change Programs in New South Wales, and Australia's National Plan to Reduce Violence Against Women and Their Children, which each call for men to be accountable and take responsibility for their actions.

In contrast to these positive trends, attitudes towards gender equity did not significantly change over program attendance. The comparatively low gender equity beliefs that existed pre-group still existed after course completion, suggesting that this risk factor was not effectively addressed, in line with Gondolf's (2000) findings. An individual's attitudes pertaining to gender equity are likely to have been developed over the course of a lifetime, so it may be the case that a single intervention program is not sufficient to challenge such deeply ingrained beliefs. Regardless, since it became apparent that gender attitudes remained stable throughout course participation, program designers have worked to address these issues more pointedly. Ongoing program evaluation and future research will explore the effectiveness of these developments.

Considering gender equity beliefs have been identified as one of the most significant predictors of behaving violently towards female partners (McGregor, 2009; Taylor \& Mouzos, 2006; Webster et al., 2014), this lack of change is an important finding. The lack of change in gender equity attitudes warrants further investigation, particularly in relation to any behavioural change. Other findings from this broader project have demonstrated changes in attitudes towards individual partners, ex-partners, and children amongst some clients, and partners and ex-partners have also reported reductions in violent behaviour alongside increased feelings of safety (Gray, Broady, Gaffney, \& Lewis, 2015). Further research is warranted to investigate the place of gender equity beliefs within these changes. In this sense, the difference between attitudes towards a partner and those towards women in general may be significant.

The complexity of relationships and attitudes in many recorded narratives, including other components of this study (Broady, Gray, Gaffney, \& Lewis, in press; Gray et al., 2016; Gray et al., 2015) and other national studies (e.g., Brown \& Flynn, 2016), indicate that significant personal change is a long-term process that only begins to emerge by the point of completing an intervention program. It may therefore be the case that the Gender Equity Scale was not sensitive enough to identify any minor changes in participants' implicit gender attitudes at this point, but that long-term positive change is possible as course participants continue their journey beyond their course attendance. Further research is needed to track men for extended periods beyond course completion to determine any long-term attitudinal change.

This finding is particularly relevant in light of the dearth of literature regarding the effectiveness of domestic violence intervention programs in addressing attitudes towards gender equity. Further research is warranted to investigate ways in which support for gender equity may be fostered amongst men who have used violence towards their partners within the context of a men's behaviour change group, particularly in light of the time limited nature of such groups. In addition, intervention programs such as that being evaluated in this study could benefit from developing strategies to directly address patriarchal attitudes and encourage greater support for gender equity, thereby shifting underlying personal beliefs that may contribute to abusive behaviour. While the current focus of such programs (e.g., taking responsibility for behaviour towards intimate partners and encouraging alternate ways of relating) appear to deliver a certain degree of success in terms of developing a personal sense of mastery, control, and self-esteem within relationships, present results indicate a lack of effectiveness in addressing underlying attitudes towards women. This may equate to a reduction in violence at a practical level (as suggested by Broady et al., 2014), while inequitable attitudes remain at a deeper level. Greater attention is therefore warranted in developing intervention programs that can effectively develop men's relationship skills and simultaneously challenge patriarchal views of gender inequality.

The combined influence each of the investigated risk factors is also worth noting. While no significant improvement in gender equity attitudes was apparent, the cumulative effect of reduced psychological distress, increased self-esteem, and increased mastery may help to mitigate the risk of violent behaviour due to low support for gender equity. While these men may retain many of their inequitable attitudes towards women, through improving their overall psychological frame of mind and developing skills to enable better ways of relating to others, the likelihood of them being triggered into 
behaving violently may well have been reduced. Considering the potential difficulties in attempting to challenge strongly held attitudes regarding gender, the cultivation of positive psychological change that was e vident in this study is argued to be a net positive result, with clear recommendations for further program development.

\subsection{Limitations}

Certain limitations of this project must be noted. Perhaps most importantly, no measure of violent behaviour was included in the surveys. Although this results in certain limitations (such as the inability to empirically examine correlations between the measured variables and violent behaviour), the focus of the wider project was to determine the extent of personal change made by course participants over time. This focus resulted in the emphasis of the survey being solely on the personal attitudes and psychological constructs outlined above. While behaviour was not measured, clinicians working with these clients do screen for violence throughout the course of the program, and have a duty of care to report any safety concerns for affected families. Although a reduction in violent behaviour is an important goal of intervention programs, the extent of participants' personal change and ability to develop positive relationship behaviours is much broader than a direct measure of violence (Westmarland \& Kelly, 2013). Future research will build on the results of this study, including an investigation of the attitudes in question and how they relate to ongoing instances of violent behaviour. In light of this limitation, it is worth noting that qualitative interviews were also conducted with partners and former partners of group participants to gain their perspectives on any behavioural changes exhibited by the men, the findings of which are beyond the scope of this paper. These findings have been published elsewhere (Gray et al., 2015), and indicate that partners of men attending Taking Responsibility often felt safer, though they described an increase in verbal abuse alongside a reduction in physical violence.

It is also acknowledged that the variables investigated in this study are not exhaustive. Due to constraints of collecting data within clinical group sessions, surveys were purposefully made as concise as possible and thus focused on select variables with previously reported clinical significance. Future research could extend the current findings with a more comprehensive investigation of salient variables. This paper also only reports on those clients who completed both preand post-group surveys. Further research analysis is warranted to investigate changes amongst those who commenced the course but did not complete it.

\subsection{Conclusion}

Despite the limitations mentioned above, positive changes were noted in relation to group members' self-esteem, mastery, and psychological distress over the time spent completing Taking Responsibility. Importantly, these changes have implications for the safety of participants' partners, children, and families. Each of these constructs has been associated with the perpetration of violent behaviour, therefore it is suggested that the positive changes evident in this study are indicators of broader personal change, including reduced violence or abuse. The apparent lack of change regarding gender equity beliefs, however, raises a significant recommendation for service improvement. Considering the strong associations between gender attitudes and domestic violence perpetration identified in previous literature, it is imperative that any intervention program for men who have used violence finds a way to effectively challenge any gender attitudes that justify, support, or promote the use of domestic violence. In doing so, women's and children's safety may be enhanced, not only through men becoming better able to control their violent actions, but also by increasing their respect towards women in general and changing perceptions of acceptable relationship behaviour.

\section{References}

ABS. (2012). Personal Safety Survey. Canberra: Australian Bureau of Statistics.

Australian Attorney-General's Department. (2010). AVERT Family Violence: Collaborative Responses in the Family Law System - Prevention strategies: Involving and engaging perpetrators. Canberra: Australian Institute of Social Relationship.

Babcock, J. C., Green, C. E., \& Robie, C. (2004). Does batterers' treatment work? A meta-analytic review of domestic violence treatment. Clinical Psychology Review, 23(8), 1023-1053. https://doi.org/10.1016/j.cpr.2002.07.001

Broady, T. R., Gray, R., \& Gaffney, I. (2014). Taking Responsibility: A psychological profile of men attending a domestic violence group work intervention program in New South Wales, Australia. Journal of Interpersonal Violence, 29(14), 2610-2629. https://doi.org/10.1177/0886260513517300

Broady, T. R., Gray, R., Gaffney, I., \& Lewis, P. (in press). 'I miss my little one a lot': How father love motivates change in men who have used violence. Child Abuse Review. https://doi.org/10.1002/car.2381

Brown, T., \& Flynn, C. (2016). Good most days: A longitudinal study of the impact of Men's Behaviour Change Programs. Paper presented at the Australian Institute of Family Studies Conference, Melbourne.

Campbell, M., Neil, J. A., Jaffe, P. G., \& Kelly, T. (2010). Engaging abusive men in seeking community intervention: A 
critical research \& practice priority. Journal of Family Violence, 25(4), 413-422. https://doi.org/10.1007/s10896-010-9302-z

COAG. (2011). National Plan to Reduce Violence against Women and their Children 2010-2022. Canberra: Council of Australian Governments.

Dardis, C. M., Dixon, K. J., Edwards, K. M., \& Turchik, J. A. (2015). An examination of the factors related to dating violence perpetration among young men and women and associated theoretical explanations: A review of the literature. Trauma, Violence, \& Abuse, 16(2), 136-152. https://doi.org/10.1177/1524838013517559

Das, M., Ghosh, S., Verma, R., O'Connor, B., Fewer, S., Virata, M. C., \& Miller, E. (2012). Gender attitudes and violence among urban adolescent boys in India. International Journal of Adolescence and Youth, 19(1), 99-112. https://doi.org/10.1080/02673843.2012.716762

Day, A., O'Leary, P., Chung, D., \& Justo, D. (2009). Domestic violence: Working with men. Sydney: The Federation Press.

Declercq, F., Willemsen, J., Audenaert, K., \& Verhaeghe, P. (2012). Psychopathy and predatory violence in homicide, violent, and sexual offences: Factor and facet relations. Legal and Criminological Psychology, 17(1), 59-74.

Gibbons, P., Collins, M., \& Reid, C. (Producer). (2011). How useful are indices of personality pathology when assessing domestic violence perpetrators?

Gondolf, E. W. (2000). How batterer program participants avoid reassault. Violence Against Women, 6(11), 1204-1222.

Gray, R. M., Broady, T. R., Gaffney, I., \& Lewis, P. (2015). How women perceive men who attend domestic violence programs. Communities, Children and Families Australia, 9(2), 71-84.

Gray, R., Broady, T., Gaffney, I., Lewis, P., Mokany, T., \& O'Neill, B. (2016). 'I'm working towards getting back together': Client accounts of motivation related to relationship status in men's behaviour change programmes in New South Wales, Australia. Child Abuse Review, 25(3), 171-182. https://doi.org/10.1002/car.2318

Hellman, C. M., Johnson, C. V., \& Dobson, T. (2010). Taking action to stop violence: A study on readiness to change among male batterers. Journal of Family Violence, 25(4), 431-438. https://doi.org/10.1007/s10896-010-9304-x

Heseltine, K., Day, A., \& Sarre, R. (2011). Prison-based correctional offender rehabilitation programs: The 2009 national picture in Australia. Canberra: Australian Institute of Criminology.

Hilton, M. F., Whiteford, H. A., Sheridan, J. S., Cleary, C. M., Chant, D. C., Wang, P. S., \& Kessler, R. C. (2008). The prevalence of psychological distress in employees and associated occupational risk factors. Journal of Occupational and Environmental Medicine, 50(7), 746-757. https://doi.org/10.1097/JOM.0b013e31817e9171

Hotaling, G. T., \& Sugarman, D. B. (1986). An analysis of risk markers in husband to wife violence: The current state of knowledge. Violence and Victims, 1(2), 101-124.

Jordan, C. E., Campbell, R., \& Follingstad, D. (2010). Violence and women's mental health: The impact of physical, sexual, and psychological aggression. Annual Review of Clinical Psychology, 6(1), 607-628. https://doi.org/10.1146/annurev-clinpsy-090209-151437

Kessler, R. C., Barker, P. R., Colpe, L. J., Epstein, J. F., Gfroerer, J. C., Hiripi, E., . . Zaslavsky, A. M. (2003). Screening for serious mental illness in the general population. Archives of General Psychiatry, 60(2), 184-189. https://doi.org/10.1001/archpsyc.60.2.184

Laing, L. (2003). What is the evidence for the effectiveness of perpetrator programmes? Sydney: Australian Domestic \& Family Violence Clearinghouse.

Lei, M.-K., Simons, R. L., Simons, L. G., \& Edmond, M. B. (2014). Gender equality and violent behavior: How neighborhood gender equality influences the gender gap in violence. Violence and Victims, 29(1), 89-108. https://doi.org/10.1891/0886-6708.VV-D-12-00102

Mackay, E., Gibson, A., Lam, H., \& Beecham, D. (2015). Perpetrator interventions in Australia: Part one - Literature review. State of knowledge paper. Sydney: Australia's National Research Organisation for Women's Safety.

McGregor, K. (2009). National Community Attitudes towards Violence Against Women Survey 2009: Project technical report. Canberra: Australian Institute of Criminology.

NSW Attorney General. (2011). Minimum standards for men's domestic violence behaviour change programs. Sydney: NSW Government.

NSW Bureau of Crime Statistics and Research. (2016). New South Wales recorded crime statistics: Quarterly update September 2016. Sydney: NSW Bureau of Crime Statistics and Research. 
Pearlin, L. I., \& Radabaugh, C. W. (1976). Economic strains and the coping function of alcohol. American Journal of Sociology, 82(3), 652-663. https://doi.org/10.1086/226357

Reingle, J. M., Jennings, W. G., Connell, N. M., Businelle, M. S., \& Chartier, K. (2014). On the pervasiveness of event-specific alcohol use, general substance use, and mental health problems as risk factors for intimate partner violence. Journal of Interpersonal Violence, 29(16), 2951-2970. https://doi.org/10.1177/0886260514527172

Rosenberg, M. (1965). Society and the adolescent self-image. Princeton, N.J.: Princeton University Press. https://doi.org/10.1515/9781400876136

Salmivalli, C. (2001). Feeling good about oneself, being bad to others? Remarks on self-esteem, hostility, and aggressive behavior. Aggression and Violent Behavior, 6(4), 375-393. https://doi.org/10.1016/S1359-1789(00)00012-4

Schmidt, M. R., Lisco, C. G., Parrott, D. J., \& Tharp, A. T. (2016). Moderating effect of negative peer group climate on the relation between men's locus of control and aggression toward intimate partners. Journal of Interpersonal Violence, 31(5), 755-773. https://doi.org/10.1177/0886260514556761

Schmitt, D. P., \& Allik, J. (2005). Simultaneous administration of the Rosenberg Self-Esteem Scale in 53 nations: Exploring the universal and culture-specific features of global self-esteem. Journal of Personality and Social Psychology, 89(4), 623-642. https://doi.org/10.1037/0022-3514.89.4.623

Sety, M. (2011). The impact of domestic violence on children: A literature review. Sydney: Australian Domestic \& Family Violence Clearinghouse.

Smith, P. H., Homish, G. G., Leonard, K. E., \& Cornelius, J. R. (2012). Intimate partner violence and specific substance use disorders: Findings from the National Epidemiologic Survey on Alcohol and Related Conditions. Psychology of Addictive Behaviors, 26(2), 236-245. https://doi.org/10.1037/a0024855

Tarzia, L., Murray, E., Humphreys, C., Glass, N., Taft, A., Valpied, J., \& Hegarty, K. (2016). I-DECIDE: An online intervention drawing on the Psychosocial Readiness Model for women experiencing domestic violence. Women's Health Issues, 26(2), 208-216. https://doi.org/10.1016/j.whi.2015.07.011

Taylor, N., \& Mouzos, J. (2006). Community Attitudes to Violence Against Women Survey 2006: A full technical report. Canberra: Australian Institute of Criminology.

Thomas, L., \& Scott-Tilley, D. (2017). Intimate partner violence: The lived experience of single women. Issues in Mental Health Nursing, 38(3), 202-211.

Tutty, L. M., \& Babins-Wagner, R. (in press). Outcomes and recidivism in mandated batterer intervention before and after introducing a specialized domestic violence court. Journal of Interpersonal Violence. https://doi.org/10.1177/0886260516647005

Walker, J. S., \& Bright, J. A. (2009). False inflated self-esteem and violence: A systematic review and cognitive model. The Journal of Forensic Psychiatry \& Psychology, 20(1), 1-32. https://doi.org/10.1080/14789940701656808

Walker, J., \& Knauer, V. (2011). Humiliation, self-esteem and violence. The Journal of Forensic Psychiatry \& Psychology, 22(5), 724-741. https://doi.org/10.1080/14789949.2011.617542

Wallinius, M., Nilsson, T., Hof vander, B., Anckarsäter, H., \& Stålenheim, G. (2012). Facets of psychopathy among mentally disordered offenders: Clinical comorbidity patterns and prediction of violent and criminal behavior. Psychiatry Research, 198(2), 279-284. https://doi.org/10.1016/j.psychres.2012.01.005

Webster, K., Pennay, D., Bricknall, R., Diemer, K., Flood, M., Powell, A., . . Ward, A. (2014). Australians' attitudes to violence against women: Full technical report, Findings from the 2013 National Community Attitudes towards Violence Against Women Survey (NCAS). Melbourne: Victorian Health Promotion Foundation.

Westmarland, N., \& Kelly, L. (2013). Why extending measurements of 'success' in domestic violence perpetrator programmes matters for social work. British Journal of Social Work, 43(6), 1092-1110. https://doi.org/10.1093/bjsw/bcs049

\section{Copyrights}

Copyright for this article is retained by the author(s), with first publication rights granted to the journal.

This is an open-access article distributed under the terms and conditions of the Creative Commons Attribution license which permits unrestricted use, distribution, and reproduction in any medium, provided the original work is properly cited. 